\title{
Utilidad del dúplex transcraneal en la Neurología: serie de casos
}

\section{Useful of transcranial duplex in neurology: case series.}

\section{Cristian Bermudez (1), Luis Fernando Roa W. (2), Camilo Romero (3), Jesús Rodríguez (4)}

\section{RESUMEN}

El ultrasonido es una herramienta ampliamente utilizada en Medicina, en diferentes áreas como ginecología, cirugía y neurología, tiene una gran variedad de indicaciones, tales como: el estudio del vasoespasmo en hemorragia subaracnoidea, cambios de flujo en estenosis intracraneana e incluso evaluar "in vivo" la actividad de algunas medidas terapéuticas. A continuación se presenta una serie de los casos más relevantes recogidos en el Hospital Universitario Mayor que fueron llevados a dúplex transcraneal y sus resultados. De igual manera una breve revisión sobre las patologías más frecuentes que se pueden encontrar mediante este estudio. El dúplex transcraneal ofrece entonces un medio diagnóstico de rápida evaluación que otorga información fehaciente para la toma de decisiones tanto en el servicio de urgencias como en hospitalización, pero se trata de una herramienta con la que se tiene poca experiencia en el país, por lo que estos hallazgos son pioneros y deben complementarse con estudios de mayor peso.

PALABRAS CLAVES: enfermedad valvular, fibrilación auricular, hemorragia subaracnoidea, trombosis intracraneal, trauma craneoencefálico, ultrasonografía (DeCS).

\author{
CONTRIBUCión DE LOS AUTORES \\ Cristian Bermudez, Diseño y Elaboración del articulo \\ Luis Fernando Roa W., Realización e interpretación de estudios ultrasonográficos \\ Camilo Romero, Realización e interpretación de estudios ultrasonográficos \\ Jesús Rodríguez, Diseño y Elaboración del articulo
}

\section{SUMMARY}

Ultrasound is a tool that is widely used in medicine in different areas such as gynecology or surgery. In neurology, it has a broad variety of indications, related to the study of vasospasm and subarachnoid haemorrhage, besides of flux changes in intracranial stenosis and it helps to evaluate the activity of some therapeutic measures. In this article we present some of the most relevant cases collected in the Hospital Universitario Mayor, wich were taken to a trans cranial duplex and we provide a short revision about most frequent pathologic that can be found through this study. The transcranial duplex offers a diagnose possibility of fast evaluation that takes reliable information to make choices in the emergency room as in the hospitalized. Although this procedure does not have many experience in the country, it is important to mention that these findings turn out to be an innovating solution to a quick diagnose that should be complemented with further information.

KEY WORDS: atrial fibrillation, craniocerebral trauma, heart valvular diseases, intracraneal thrombosis, subarachnoid hemorrhage, ultrasonography $(\mathrm{MeSH})$.

(1) Medico Hospitalario Neurología. Hospital Universitario mederi.

(2) Neurología vascular, Epidemiología clínica. Hospital Universitario mederi.

(3) Neurología vascular. Fundación Universitaria Cárdio-infantil.

(4) Neurología. Neurofisiología. Fundación Universitaria Cárdio-infantil.

Recibido 3/08/16. Aceptado: 4/07/17.

Correspondencia: Cristian Bermudez, restart1290@hotmail.com

94 


\section{INTRODUCCIÓN}

El ultrasonido se ha convertido en una herramienta eficiente en el estudio de la enfermedad cerebrovascular isquémica y/o hemorrágica en la neurología. Es un examen útil para la tamización de la estenosis intracraneal, presencia de oclusión distal, síndrome de robo de la subclavia, evaluación de la vasorreactividad cerebral, monitorización de vasoespasmo en la hemorragia subaracnoidea, detección en el shunt derecha a izquierda (microembolismos), anemia drepanocitica, aumento de la presión intracraneal, arresto circulatorio, muerte cerebral, monitorización de la endarterectomía, malformaciones arteriovenosas; y por último como seguimiento y/o efecto terapéutico en la recanalización arterial durante la trombolisis en el infarto cerebral agudo (1-6).

Objetivo: describir una serie de pacientes con enfermedad cerebrovascular isquémica y hemorrágica con hallazgos anormales en el dúplex transcraneal (DTC) y hacer una breve revisión sobre las patologías evidenciadas y sus hallazgos típicos en este tipo de estudios.

Materiales y métodos: se realizó una serie de casos de pacientes con enfermedad cerebrovascular a quienes se les realizó dúplex transcraneal, entre noviembre 2015 y enero de 2016. Se presentan los hallazgos clínicos más relevantes tanto ultrasonográficos como imagenológicos.

Resultados: se incluyeron 6 casos con diferentes alteraciones en la morfología, velocidades picosistólicas, medias e índice de pulsatilidad como ejemplo de las patologías que pueden estudiarse a través del DTC.

\section{Presentación de casos}

Caso 1: masculino de 32 años, afasia y hemiparesia derecha con diagnóstico de infarto cerebral en arteria cerebral media izquierda (figura 1a). DTC: disminución de sístole/ diástole, velocidades medias e índice de pulsatilidad $(<1,2)$ de la arteria cerebral media izquierda con un patrón oclusivo TIBI II (flujo amortiguado o aplanado) (figura $1 \mathrm{~b}$ ).

Caso 2: masculino de 76 años, infarto agudo de miocardio y alteración de la conciencia con diagnóstico de infarto pontino derecho (figura 2a). DTC: aumento de los índices de pulsatilidad con disminución brusca y acortamiento de flujo diastólico en arteria vertebral derecha (patrón preestenótico) (figura 2b).

Caso 3: femenino de 98 años, disartria severa. DTC: disminución del índice de pulsatilidad con presencia de inversión de la onda y flujos retrógrados en la arteria vertebral derecha (síndrome de robo vertebral) (figura 3).

Caso 4: femenino de 56 años, amnesia global transitoria y amaurosis fugax. valvulopatía aórtica. DTC: velocidades picosistolicas disminuidas, velocidades medias disminuidas y ondas dicróticas, con pulso "bisferiens" (enfermedad valvular aórtica (estenosis y/o insuficiencia), cardiomiopatía hipertrófica obstructiva) (figura 4).

Caso 5: masculino de 66 años, trauma cráneo encefálico moderado con hemorragia subaracnoidea (figura 5a). DTC: aumento de velocidades picosistolicas de arteria cerebral media izquierda, sugestivo de hiperemia sin vasoespasmo (figura 5b).
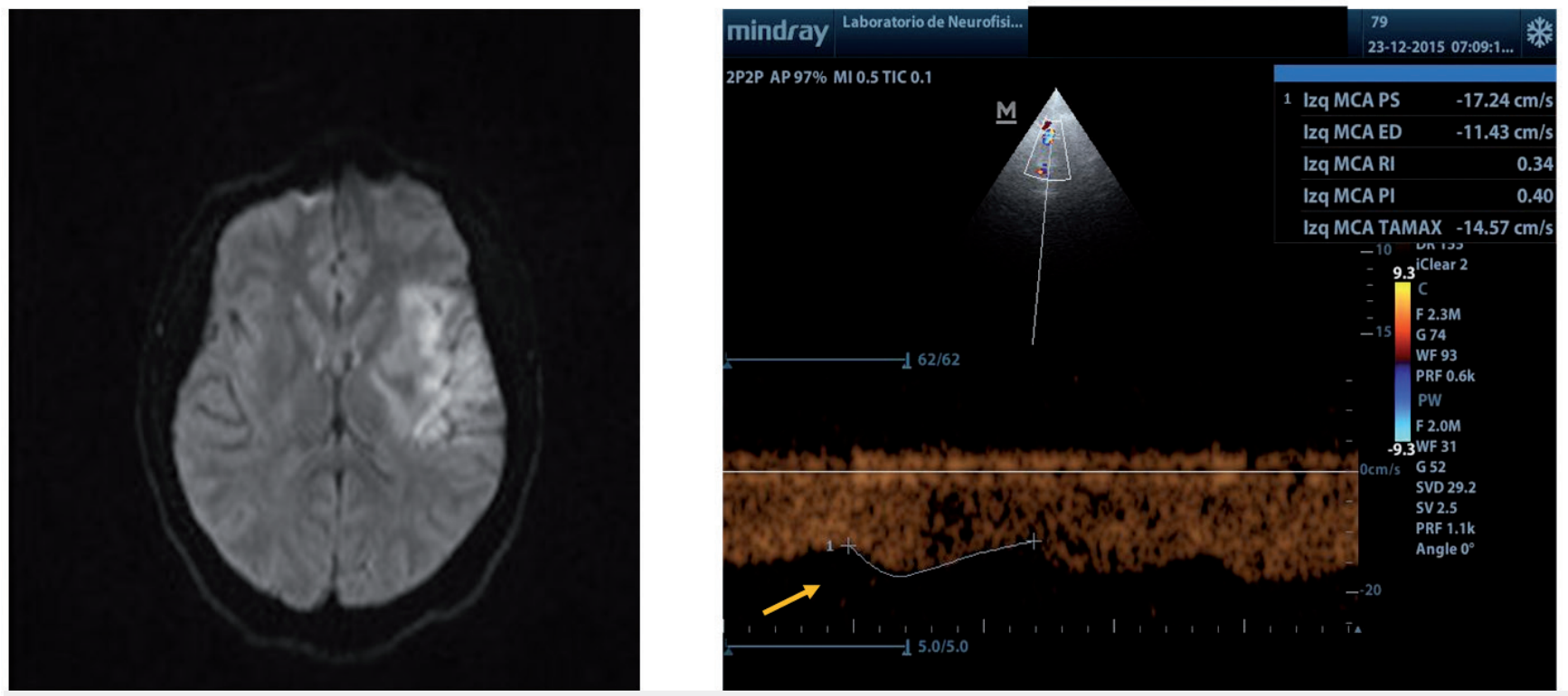

Figura 1a. RMN cerebral simple: lesión isquémica en ACMI; 1b. Flecha: ver disminución de velocidad picosistolica y disminución de velocidades medias. 

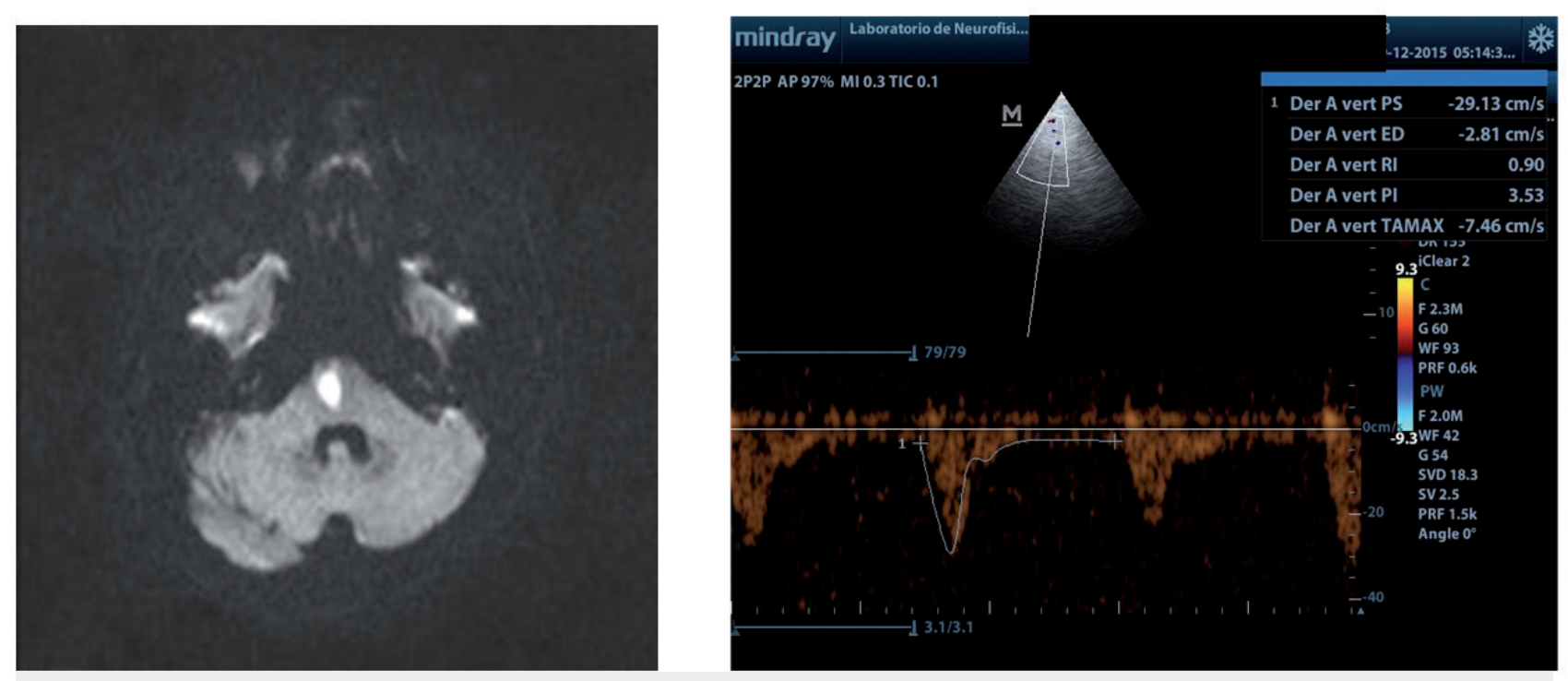

Figura 2a. RMN cerebral simple: lesión isquémica pontina derecha; 2 b. Flecha: nótese disminución rápida de la onda sistólica con poco flujo en diástole.

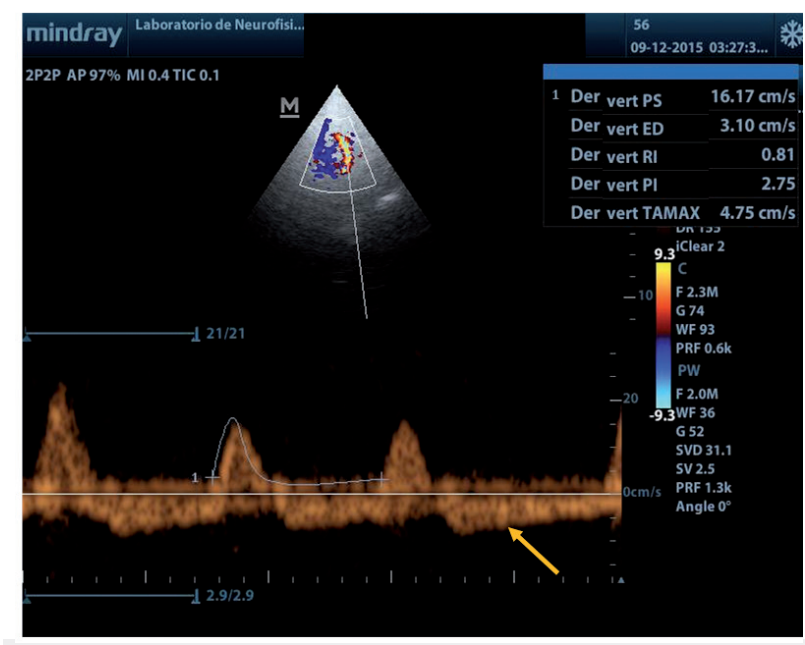

Figura 3. Dúplex transcraneal en el que se evidencia inversión de flujo en diástole.

Caso 6: masculino de 71 años, hemiparesia derecha y disartria transitorias, sin factores de riesgo cerebrovascular. DTC: ondas arrítmicas e irregulares (figura 6a). EKG: fibrilación auricular (figura 6b).

Discusión: en los casos previamente descritos se evidenciaron las alteraciones más frecuentes que se pueden encontrar en un grupo de patologías con influencia directa en el día a día de la práctica de neurología.

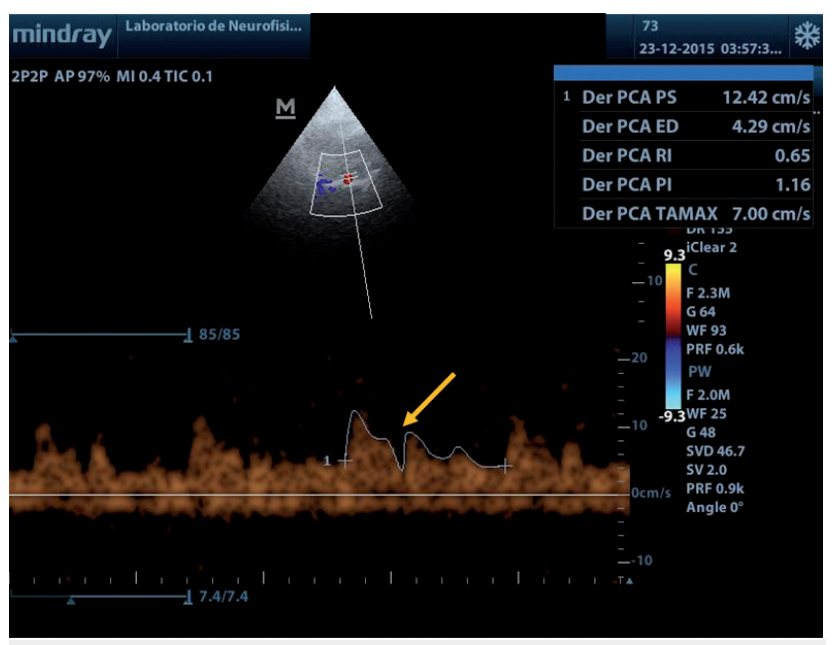

Figura 4. Dúplex transcraneal flecha: se evidencia onda dicrotica.

\section{ESTENOSIS INTRACRANEANA}

La estenosis intracraneana corresponde a la estrechez de la luz de los vasos intracerebrales por diferentes causas, siendo la ateroesclerosis la más frecuente (7) considerada como uno de los principales factores de riesgo para la enfermedad cerebrovascular. En la actualidad se cuenta con una variedad de métodos diagnósticos que funcionan como herramientas útiles para su estadificación, sin embargo, los 

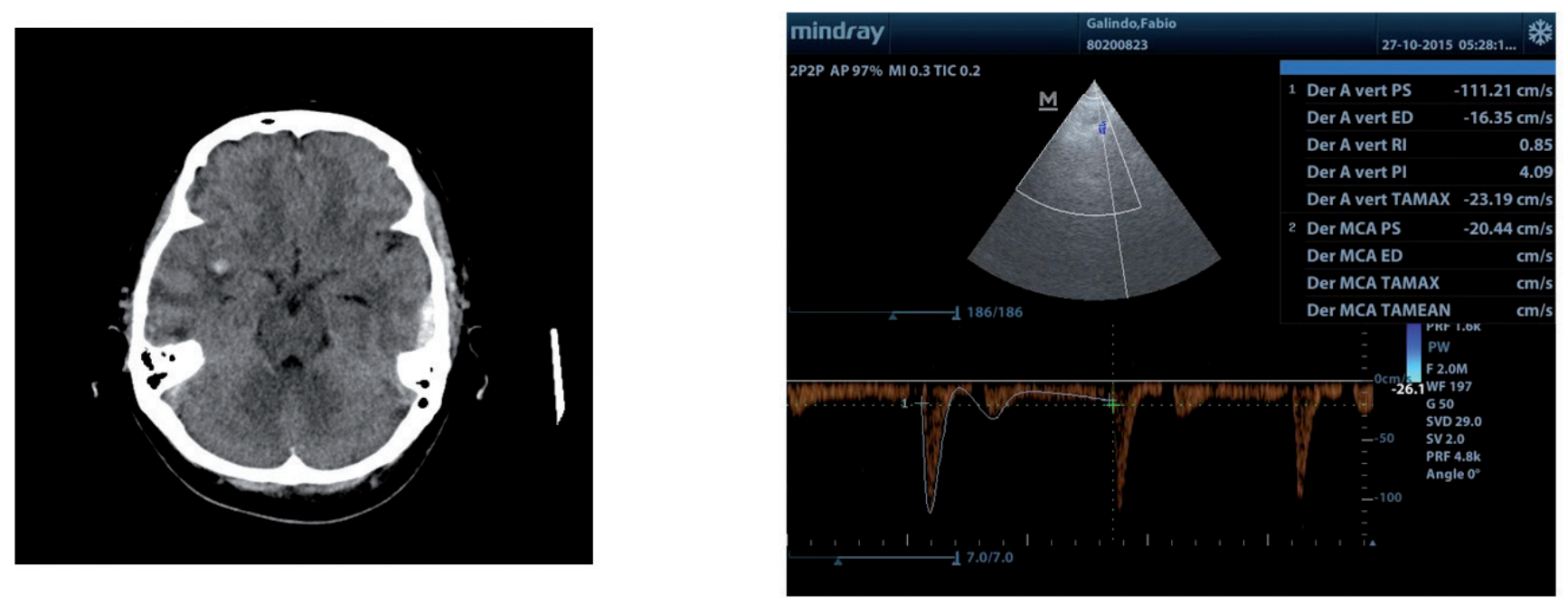

Figura 5a. TC de cráneo simple evidenciando lesión hemorrágica a nivel temporoparietal. 5b. DTC: ver aumento de velocidades picosistólicas en arteria vertebral derecha.
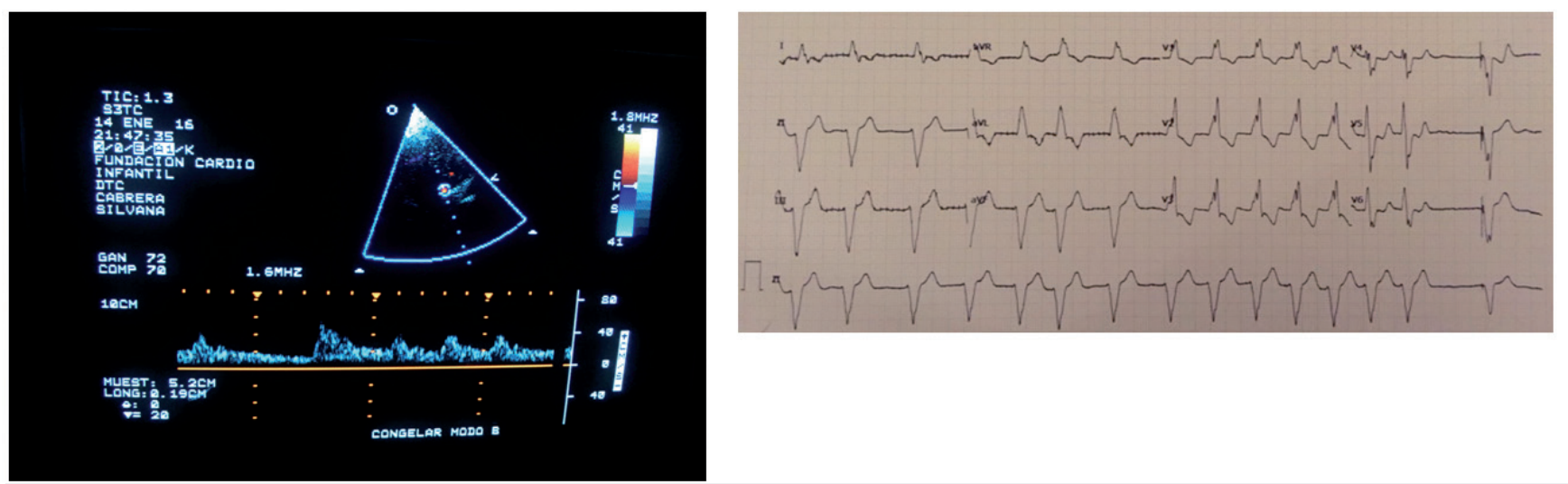

Figura 6a.Dúplex transcraneal que demuestran latidos irregulares; 6b. Ver segmento R-R irregular.

métodos poco invasivos como el DTC pueden resultar más útiles y cómodos al evaluar directamente la integridad de las paredes del vaso sanguíneo y no sólo su luz.

\section{FIBRILACIÓN AURICULAR}

Por su parte, la fibrilación auricular es la arritmia cardiaca más frecuente en el mundo, se cree que afecta al 1 $\%$ de la población y hasta en un $8 \%$ a los mayores de 80 años $(8,9)$. Es ampliamente reconocida como un factor de riesgo cerebrovascular por su potencial cardioembólico, sin embargo, en muchos pacientes se caracteriza por una cualidad paroxística rara vez evidenciada en trazados electrocardiográficos o durante el examen físico (10), por lo que cualquier estudio, sea invasivo, eléctrico o semiológico que permita su diagnóstico representa una gran ayuda, como lo es el dúplex transcraneal.

\section{Hemorragia subaracnoidea}

Por otro lado, la hemorragia subaracnoidea, traumática o aneurismática, representa una condición de alto riesgo, tanto por la lesión inicial como por el vasoespasmo sintomático, por lo que el cuidado multidisciplinario de este grupo de pacientes es vital para la aproximación adecuada en la unidad de cuidado intensivo (11).

El vasoespasmo es evidente en al menos el $70 \%$ de las hemorragias subaracnoideas de origen aneurismático, pero solo del 20 al $40 \%$ de los casos desarrollarán un nuevo déficit neurológico asociado al mismo (12), por lo que 
contar con las herramientas necesarias para su diagnóstico oportuno es vital.

Pese a las variaciones interobservador y las limitaciones que existen en la circulación posterior, el dúplex transcraneal se mantiene como una herramienta de alta confiabilidad en la evaluación del vasoespasmo en la arteria cerebral media (13).

\section{CONCLUSIONES}

Mediante los casos ilustrados documentamos, la utilidad del dúplex transcraneal como estudio de tamización en la enfermedad cerebrovascular y otras patologías, así como la ventaja que representa al ser realizado en relación directa con el neurólogo y la capacidad de la correlación clínica del mismo. Al tratarse de una herramienta con la que pocos centros de Colombia cuentan, los resultados obtenidos son pioneros en el estudio de nuestra población.

\section{Conflictos de intereses}

Los autores declaran que el estudio fue realizado sin ningún tipo de relación comercial o relaciones financiarías que pudieran ser consideradas como conflicto de intereses.

\section{REFERENCIAS}

1. Demchuk AM, Burgin WS, Christou I, Felberg RA, Barber PA, Hill MD, et al. Thrombolysis in brain ischemia (TIBI) transcranial doppler flow grades predict clearly recovery and mortality in patients treated with tissue plasminogen activator. Stroke. 2001;32(1):89-93.

2. Brunser A, Lavados PM, Hoppe A, López J, Valenzuela M, Rivas R. Accuracy of trnascranial Doppler compared with CT angiography in dignosing arterial obstructions in acute ischemic strokes. Stroke. 2009;40(6):2037-41. http://dx.doi. org/10.1161/STROKEAHA.108.542704.

3. Baumgartner RW, Mattle P, Schroth G. Assessment of $\geq 50 \%$ and $<50 \%$ intracranial stenoses by transcranial color-coded duplex sonography. Stroke.1999;30(1):87-92.

4. Gauss M, Zanette E. Detection of right-to-left shunt with ultrasound contrast agent and transcranial doppler sonography. Cerebrovasc Dis. 2000;10(6):490-96.

5. Alexandrov AV, Demchuk AM, Burgin WS, Robinson DJ, Grotta J. CCLOTBUST Investigators. Ultrasound-enhanced thrombolysis for acute ischemic stroke: phase I. Findings of the CLOTBUST trial. J Neuroimaging. 2004;14(2):113-117.

6. Arenas BW, Silva FA, Zarruk JG, Romero J, Ruiz NP, Cárdenas N. Utilidad del doppler transcraneal en enfermedad cerebrovascular crítica. Acta Neurol Colomb. 2007;23(4):266-277.

7. Carvalho M, Oliveira A, Azevedo E, Bastos-Leite AJ. Intracranial arterial stenosis. J Stroke Cerebrovasc Dis. 2014;23(4):599609.

8. Hahne K, Mönnig G, Samol A. Atrial fibrillation and silent stroke: links, risks, and challenges. Vasc Health Risk Manag.
2016;12:65-74. . http://dx.doi.org/10.2147/VHRM.S81807

9. January CT, Wann L, Alpert JS, Calkins H, Cigarroa JE, Cleveland JC Jr, et al. 2014 AHA/ACC/HRS guideline for the management of patients with atrial fibrillation: executive summary: A Report of the American College of Cardiology/American Heart Association task force on practice guidelines and the Heart Rhythm Society. J Am Coll Cardiol. 2014;64(21):22462280. http://dx.doi.org/10.1161/CIR.0000000000000040

10. Alhadramy O, Jeerakathil TJ, Majumdar SR, Najjar E, Choy J, Saqqur M. Prevalence and predictors of paroxysmal atrial fibrillation on Holter monitor in patients with stroke or transient ischemic attack. Stroke. 2010;41(11):2596-600. http://dx.doi. org/10.1161/STROKEAHA.109.570382

11. Bauer AM, Rasmussen PA, Treatment of intracranial vasospasm following subarachnoid hemorrhage. Front Neurol. 2014;20;5:72. http://dx.doi.org/ 10.3389/fneur.2014.00072

12. Al-Tamimi YZ, Orsi NM, Quinn AC, Homer-Vanniasinkam S, Ross SA. A review of delayed ischemic neurologic deficit following aneurysmal subarachnoid hemorrhage: historical overview, current treatment, and pathophysiology. World Neurosurg. 2010;73(6):654-67. http://dx.doi.org/10.1016/j. wneu.2010.02.005.

13. Washington CW, Zipfel GJ. Participants in the international multi-disciplinary consensus conference on the critical care management of subarachnoid hemorrhage. Detection and monitoring of vasospasm and delayed cerebral ischemia: a review and assessment of the literature. Neurocrit Care. 2011; 15(2):312-7. http://dx.doi.org/10.1007/s12028-011-9594-8 